\title{
Asymptotic Behavior of Solutions to a Linear Volterra Integrodifferential System
}

\author{
Yue-Wen Cheng and Hui-Sheng Ding \\ College of Mathematics and Information Science, Jiangxi Normal University, Nanchang, Jiangxi 330022, China \\ Correspondence should be addressed to Hui-Sheng Ding; dinghs@mail.ustc.edu.cn
}

Received 24 July 2013; Accepted 18 September 2013

Academic Editor: Josef Diblík

Copyright ( 2013 Y.-W. Cheng and H.-S. Ding. This is an open access article distributed under the Creative Commons Attribution License, which permits unrestricted use, distribution, and reproduction in any medium, provided the original work is properly cited.

We investigate the asymptotic behavior of solutions to a linear Volterra integrodifferential system $x_{i}^{\prime}(t)=a_{i}(t)+b_{i}(t) x_{i}(t)+$ $\sum_{j=1}^{n} \int_{0}^{t} K_{i j}(t, s) x_{j}(s) d s, t \in \mathbb{R}^{+}, i=1,2, \ldots, n$. We show that under some suitable conditions, there exists a solution for the above integrodifferential system, which is asymptotically equivalent to some given functions. Two examples are given to illustrate our theorem.

\section{Introduction}

Throughout this paper, we denote by $\mathbb{N}$ the set of positive integers, by $\mathbb{R}$ the set of all real numbers, by $\mathbb{R}^{+}$the set of all nonnegative real numbers, and by $\mathbb{R}^{n}$ the set of all $n$ dimensional real vectors. Moreover, $B C\left(\mathbb{R}^{+}, \mathbb{R}^{n}\right)$ denotes the Banach space of all bounded and continuous functions $f$ : $\mathbb{R}^{+} \rightarrow \mathbb{R}^{n}$ with the norm

$$
\|f\|=\sup _{t \in \mathbb{R}^{+}} \max \left|f_{j}(t)\right|,
$$

where $f(t)=\left(f_{1}(t), \ldots, f_{n}(t)\right)^{T}$ for $t \in \mathbb{R}^{+}$.

The aim of this paper is to study some asymptotic behavior of solutions to the following linear Volterra integrodifferential system:

$$
\begin{array}{r}
x_{i}^{\prime}(t)=a_{i}(t)+b_{i}(t) x_{i}(t)+\sum_{j=1}^{n} \int_{0}^{t} K_{i j}(t, s) x_{j}(s) d s, \\
t \in \mathbb{R}^{+}, i=1,2, \ldots, n,
\end{array}
$$

where $a_{i}, b_{i}: \mathbb{R}^{+} \rightarrow \mathbb{R}$ and $K_{i j}: \mathbb{R}^{+} \times \mathbb{R}^{+} \rightarrow \mathbb{R}, i, j=1,2$, $\ldots, n$ are all continuous functions.

Definition 1. We call $x=\left(x_{1}, x_{2}, \ldots, x_{n}\right)^{T}: \mathbb{R}^{+} \rightarrow \mathbb{R}^{n}$ a solution of system (2) if $x$ is continuously differentiable and satisfies (2).
The asymptotic behavior of solutions has been an important and interesting topic in the qualitative theory of differential and difference equations. Especially, recently, many authors have made interesting and important contributions on the asymptotic behavior of solutions for Volterra type difference equations (e.g., we refer the reader to [1-10] and references therein).

Very recently, Diblík and Schmeidel [6] obtained a very interesting result concerning the asymptotic behavior of solutions for the following linear Volterra difference equation:

$$
x(n+1)=a(n)+b(n) x(n)+\sum_{i=0}^{n} K(n, i) x(i) .
$$

More specifically, they proved that for every admissible constant $c \in \mathbb{R}$, there exists a solution $x=x(n)$ of (3) such that

$$
x(n) \sim\left(c+\sum_{i=0}^{n-1} \frac{a(i)}{\beta(i+1)}\right) \beta(n), \quad n \longrightarrow \infty,
$$

where $\beta(n)=\prod_{i=0}^{n-1} b(i)$. However, to the best of our knowledge, it seems that there is no literature concerning such asymptotic behavior of solutions for Volterra type differential equations. That is the main motivation of this paper. In this paper, we will adopt the idea in the proof of [6] to investigate 
some asymptotic behaviors of solutions for Volterra differential system (2).

\section{Main Result}

Before establishing our main result, we first give an "ArzelaAscoli" type theorem for the subsets of $B C\left(\mathbb{R}^{+}, \mathbb{R}^{n}\right)$.

Lemma 2. Let $\mathscr{F} \subset B C\left(\mathbb{R}^{+}, \mathbb{R}^{n}\right)$, satisfying (i) $\mathscr{F}$ is uniformly bounded; (ii) $\mathscr{F}$ is equiuniformly continuous on every compact subset of $\mathbb{R}^{+}$; (iii) for every $\varepsilon>0$, there exist $f_{\varepsilon} \in B C\left(\mathbb{R}^{+}, \mathbb{R}^{n}\right)$ and $T_{\varepsilon}>0$ such that $\left\|f(t)-f_{\varepsilon}(t)\right\|<\varepsilon$ for all $f \in \mathscr{F}$ and $t \geq T_{\varepsilon}$. Then $\mathscr{F}$ is precompact in $B C\left(\mathbb{R}^{+}, \mathbb{R}^{n}\right)$.

Proof. By the condition (iii), for every $k \in \mathbb{N}$, there exist $T_{k}>$ 0 such that

$$
\left\|F_{1}(t)-F_{2}(t)\right\|<\frac{1}{k}
$$

for all $F_{1}, F_{2} \in \mathscr{F}$ and $t \geq T_{k}$.

Let $\left\{f_{n}\right\}$ be a sequence in $\mathscr{F}$. By (i) and (ii), it follows from Arzela-Ascoli theorem that for every $k \in \mathbb{N}$, there exists a subsequence $\left\{f_{n}^{k}\right\} \subset\left\{f_{n}\right\}$ such that $\left\{f_{n}^{k}\right\}$ is uniformly convergent on $\left[0, T_{k}\right]$. Then, by choosing the diagonal sequence, we can get a subsequence $\left\{f_{m}\right\} \subset\left\{f_{n}\right\}$ such that, for every $k \in \mathbb{N},\left\{f_{m}\right\}$ is uniformly convergent on $\left[0, T_{k}\right]$.

It remains to show that $\left\{f_{m}\right\}$ is uniformly convergent on $\mathbb{R}^{+}$. For every $\varepsilon>0$, choose $k_{0} \in \mathbb{N}$ with $1 / k_{0}<\varepsilon$. Since $\left\{f_{m}\right\}$ is uniformly convergent on $\left[0, T_{k_{0}}\right]$, for the above $\varepsilon>0$, there exists $N \in \mathbb{N}$ such that

$$
\sup _{t \in\left[0, T_{k_{0}}\right]}\left\|f_{m_{1}}(t)-f_{m_{2}}(t)\right\| \leq \varepsilon
$$

for all $m_{1}, m_{2} \geq N$; Combining this with (5), we conclude that

$$
\sup _{t \in \mathbb{R}^{+}}\left\|f_{m_{1}}(t)-f_{m_{2}}(t)\right\| \leq \varepsilon
$$

for all $m_{1}, m_{2} \geq N$, that is, $\left\{f_{m}\right\}$ is uniformly convergent on $\mathbb{R}^{+}$. This completes the proof.

Throughout the rest of this paper, for every $i \in\{1,2, \ldots$, $n\}$, we assume that

$$
A_{i}=\sup _{t \in \mathbb{R}^{+}}\left|A_{i}(t)\right|<+\infty,
$$

where

$$
\begin{gathered}
A_{i}(t)=\int_{0}^{t} \frac{a_{i}(s)}{\beta_{i}(s)} d s \\
\beta_{i}(t)=\exp \left(\int_{0}^{t} b_{i}(s) d s\right), \quad t \in \mathbb{R}^{+}, \\
0 \leq M_{i}:=\sum_{j=1}^{n} \int_{0}^{+\infty}\left(\int_{0}^{t}\left|K_{i j}(t, s) \frac{\beta_{j}(s)}{\beta_{i}(t)}\right| d s\right) d t<1 .
\end{gathered}
$$

Theorem 3. Assume that

$$
\begin{array}{r}
0<\liminf _{t \rightarrow+\infty} \beta_{i}(t) \leq \limsup _{t \rightarrow+\infty} \beta_{i}(t)<+\infty, \\
i=1,2, \ldots, n .
\end{array}
$$

Let $c=\left(c_{1}, c_{2}, \ldots, c_{n}\right)^{T} \in \mathbb{R}^{n}$ with $c_{i}+A_{i}(t) \geq 0$ for all $i \in\{1,2, \ldots, n\}$ and $t \in \mathbb{R}^{+}$. Then, there exists a solution $x=$ $\left(x_{1}, x_{2}, \ldots, x_{n}\right)^{T}: \mathbb{R}^{+} \rightarrow \mathbb{R}^{n}$ of system (2) such that

$$
x_{i}(t) \sim\left(c_{i}+A_{i}(t)\right) \beta_{i}(t), \quad t \longrightarrow+\infty, i=1,2, \ldots, n,
$$

provided that $\liminf _{t \rightarrow+\infty}\left(c_{i}+A_{i}(t)\right)>0$.

Proof. We define that

$$
\begin{gathered}
\alpha_{i}(0)=\frac{\left(c_{i}+A_{i}\right) M_{i}}{1-M_{i}}, \\
\alpha_{i}(t)=\left(c_{i}+A_{i}+\alpha_{i}(0)\right) \\
\times \sum_{j=1}^{n} \int_{t}^{+\infty}\left(\int_{0}^{s}\left|K_{i j}(s, p) \frac{\beta_{j}(p)}{\beta_{i}(s)}\right| d p\right) d s,
\end{gathered}
$$

for all $i \in\{1,2, \ldots, n\}$ and $t \in \mathbb{R}^{+}$. Moreover, we define an operator $\rho=\left(\rho_{1}, \rho_{2}, \ldots, \rho_{n}\right)^{T}$ on

$$
\begin{gathered}
S=\left\{z \in B C\left(\mathbb{R}^{+}, \mathbb{R}^{n}\right): c_{i}+A_{i}(t)-\alpha_{i}(0)\right. \\
\leq z_{i}(t) \leq c_{i}+A_{i}(t)+\alpha_{i}(0), \\
\left.i=1,2, \ldots, n, t \in \mathbb{R}^{+}\right\}
\end{gathered}
$$

by

$$
\begin{aligned}
\left(\rho_{i} z\right)(t)= & c_{i}+A_{i}(t) \\
& -\sum_{j=1}^{n} \int_{t}^{+\infty}\left(\int_{0}^{s} K_{i j}(s, p) z_{j}(p) \frac{\beta_{j}(p)}{\beta_{i}(s)} d p\right) d s, \\
& i=1,2 \ldots, n,
\end{aligned}
$$

for $t \in \mathbb{R}^{+}$and $z \in S$. It is easy to see that $S$ is a nonempty, closed, and convex set in $B C\left(\mathbb{R}^{+}, \mathbb{R}^{n}\right)$. Next, we divide the remaining proof into two steps.

Step 1. $\rho(S) \subset S, \rho$ is continuous, and $\overline{\rho(S)}$ is compact.

Let $z \in S$. We have

$$
\begin{array}{r}
\left|\left(\rho_{i} z\right)(t)\right| \leq\left|c_{i}\right|+A_{i}+M_{i} \cdot\|z\|<+\infty, \\
t \in \mathbb{R}^{+}, \quad i=1,2, \ldots, n .
\end{array}
$$

In addition, since $z \in S$, we have

$$
\left|z_{i}(t)\right| \leq c_{i}+A_{i}+\alpha_{i}(0), \quad t \in \mathbb{R}^{+}, i=1,2, \ldots, n .
$$


Then, it follows that

$$
\begin{aligned}
& \left|\left(\rho_{i} z\right)(t)-\left(c_{i}+A_{i}(t)\right)\right| \\
& \quad \leq \sum_{j=1}^{n} \int_{t}^{+\infty}\left(\int_{0}^{s}\left|K_{i j}(s, p) z_{j}(p) \frac{\beta_{j}(p)}{\beta_{i}(s)}\right| d p\right) d s \\
& \quad \leq\left[c_{i}+A_{i}+\alpha_{i}(0)\right] \cdot M_{i}=\alpha_{i}(0)
\end{aligned}
$$

for all $i \in\{1,2, \ldots, n\}$ and $t \in \mathbb{R}^{+}$. Thus, we conclude that $\rho(S) \subset S$.

For every $\varepsilon>0$, there exists a constant $\delta=\varepsilon / \max _{1 \leq i \leq n} M_{i}$ such that for all $z, y \in S$ with $\|z-y\|<\delta$, we have

$$
\begin{aligned}
&\left|\left(\rho_{i} z\right)(t)-\left(\rho_{i} y\right)(t)\right| \\
&=\mid \sum_{j=1}^{n} \int_{t}^{+\infty}\left(\int_{0}^{s} K_{i j}(s, p) z_{j}(p) \frac{\beta_{j}(p)}{\beta_{i}(s)} d p\right) d s \\
& \quad-\sum_{j=1}^{n} \int_{t}^{+\infty}\left(\int_{0}^{s} K_{i j}(s, p) y_{j}(p) \frac{\beta_{j}(p)}{\beta_{i}(s)} d p\right) d s \mid \\
& \leq \delta \cdot M_{i} \leq \varepsilon, \quad i \in\{1,2, \ldots, n\}, t \in \mathbb{R}^{+},
\end{aligned}
$$

which means that $\rho$ is continuous.

Next, we show that $\rho(S)$ is precompact. Firstly, for every $x \in S$, we have

$$
\begin{aligned}
\|\rho x\| & =\sup _{t \in \mathbb{R}^{+1}} \max \left|\left(\rho_{i} x\right)(t)\right| \\
& \leq \max _{1 \leq i \leq n}\left[c_{i}+A_{i}+\alpha_{i}(0)\right],
\end{aligned}
$$

which means that $\rho(S)$ is uniformly bounded. Secondly, for every $z \in S, t_{1}, t_{2} \in \mathbb{R}^{+}$and $i=1,2, \ldots, n$, we have

$$
\begin{aligned}
& \left|\left(\rho_{i} z\right)\left(t_{1}\right)-\left(\rho_{i} z\right)\left(t_{2}\right)\right| \\
& =\mid \sum_{j=1}^{n} \int_{t_{1}}^{+\infty}\left(\int_{0}^{s} K_{i j}(s, p) z_{j}(p) \frac{\beta_{j}(p)}{\beta_{i}(s)} d p\right) d s \\
& \quad-\sum_{j=1}^{n} \int_{t_{2}}^{+\infty}\left(\int_{0}^{s} K_{i j}(s, p) z_{j}(p) \frac{\beta_{j}(p)}{\beta_{i}(s)} d p\right) d s \mid \\
& \leq \max _{1 \leq i \leq n}\left[c_{i}+A_{i}+\alpha_{i}(0)\right] \\
& \quad\left|\sum_{j=1}^{n} \int_{t_{1}}^{t_{2}}\left(\int_{0}^{s}\left|K_{i j}(s, p) \frac{\beta_{j}(p)}{\beta_{i}(s)}\right| d p\right) d s\right|,
\end{aligned}
$$

which yields that $\rho(S)$ is equiuniformly continuous on every compact subsets of $\mathbb{R}^{+}$. Thirdly, by the definition of $M_{i}$, for every $\varepsilon>0$, there exists $T>0$ such that for all $t \geq T$ and $z \in S$, we have

$$
\begin{aligned}
\sum_{j=1}^{n} \int_{t}^{+\infty}\left(\int_{0}^{s}\left|K_{i j}(s, p) \frac{\beta_{j}(p)}{\beta_{i}(s)}\right| d p\right) d s & \frac{\varepsilon}{} \quad<\frac{\max _{1 \leq i \leq n}\left[c_{i}+A_{i}+\alpha_{i}(0)\right]}{\operatorname{man}}, \quad i=1,2, \ldots, n,
\end{aligned}
$$

which yields that

$$
\left\|\rho_{i} z-\rho_{i} 0\right\|<\varepsilon, \quad i=1,2, \ldots, n,
$$

and thus $\|\rho z-\rho 0\|<\varepsilon$. Then, by Lemma 2, we know that $\rho(S)$ is precompact.

Step 2. By Step 1 and Schauder's fixed-point theorem, $\rho$ has a fixed point in $S$; that is, there exists $z^{0}=\left(z_{1}^{0}, z_{2}^{0}, \ldots, z_{n}^{0}\right)^{T} \in S$ such that

$$
\begin{aligned}
z_{i}^{0}(t)= & c_{i}+A_{i}(t) \\
& -\sum_{j=1}^{n} \int_{t}^{+\infty}\left(\int_{0}^{s} K_{i j}(s, p) z_{j}^{0}(p) \frac{\beta_{j}(p)}{\beta_{i}(s)} d p\right) d s,
\end{aligned}
$$

for all $i \in\{1,2, \ldots, n\}$ and $t \in \mathbb{R}^{+}$. Noting that

$$
\sup _{z \in S}\|z\| \leq \max _{1 \leq i \leq n}\left[c_{i}+A_{i}+\alpha_{i}(0)\right],
$$

we have

$$
\begin{aligned}
\mid z_{i}^{0}(t) & -\left(c_{i}+A_{i}(t)\right) \mid \\
\leq & \max _{1 \leq i \leq n}\left[c_{i}+A_{i}+\alpha_{i}(0)\right] \\
& \cdot \sum_{j=1}^{n} \int_{t}^{+\infty}\left(\int_{0}^{s}\left|K_{i j}(s, p) \frac{\beta_{j}(p)}{\beta_{i}(s)}\right| d p\right) d s,
\end{aligned}
$$

for all $i \in\{1,2, \ldots, n\}$ and $t \in \mathbb{R}^{+}$. Then, it is easy to see that

$$
\lim _{t \rightarrow+\infty}\left|z_{i}^{0}(t)-\left(c_{i}+A_{i}(t)\right)\right|=0, \quad i=1,2, \ldots, n,
$$

Combining this with

$$
\liminf _{t \rightarrow+\infty}\left(c_{i}+A_{i}(t)\right)>0,
$$

we have

$$
\lim _{t \rightarrow+\infty} \frac{z_{i}^{0}(t)}{c_{i}+A_{i}(t)}=1, \quad i=1,2, \ldots, n
$$

that is,

$$
z_{i}^{0}(t) \sim c_{i}+A_{i}(t), \quad t \longrightarrow+\infty, i=1,2, \ldots, n .
$$
by

Now, define a function $x=\left(x_{1}, x_{2}, \ldots, x_{n}\right)^{T}: \mathbb{R}^{+} \rightarrow \mathbb{R}^{n}$ $x_{i}(t)=z_{i}^{0}(t) \beta_{i}(t), \quad i=1,2, \ldots, n, t \in \mathbb{R}^{+}$. 
It follows from (23) that

$$
\begin{array}{r}
\frac{d}{d t} z_{i}^{0}(t) \\
=\frac{a_{i}(t)}{\beta_{i}(t)}+\sum_{j=1}^{n} \int_{0}^{t} K_{i j}(t, p) z_{j}^{0}(p) \frac{\beta_{j}(p)}{\beta_{i}(t)} d p, \\
\quad i=1,2, \ldots, n, \quad t \in \mathbb{R}^{+},
\end{array}
$$

which yields that

$$
\begin{aligned}
& \frac{x_{i}^{\prime}(t) \beta_{i}(t)-x_{i}(t) \beta_{i}^{\prime}(t)}{\beta_{i}^{2}(t)} \\
& =\frac{a_{i}(t)}{\beta_{i}(t)}+\sum_{j=1}^{n} \int_{0}^{t} K_{i j}(t, s) \frac{x_{j}(s)}{\beta_{i}(t)} d s, \\
& \quad i=1,2, \ldots, n, \quad t \in \mathbb{R}^{+} .
\end{aligned}
$$

Then, we get

$$
\begin{aligned}
x_{i}^{\prime}(t)= & a_{i}(t)+b_{i}(t) x_{i}(t) \\
+ & \sum_{j=1}^{n} \int_{0}^{t} K_{i j}(t, s) x_{j}(s) d s, \\
& \quad i=1,2, \ldots, n, \quad t \in \mathbb{R}^{+},
\end{aligned}
$$

which means that $x$ is a solution to system (2). In addition, combining (28) with the assumption

$$
\begin{array}{r}
0<\liminf _{t \rightarrow+\infty} \beta_{i}(t) \leq \limsup _{t \rightarrow+\infty} \beta_{i}(t)<+\infty, \\
i=1,2, \ldots, n,
\end{array}
$$

we get

$$
\lim _{t \rightarrow+\infty} \frac{x_{i}(t)}{\left(c_{i}+A_{i}(t)\right) \beta_{i}(t)}=1, \quad i=1,2, \ldots, n,
$$

which yields (11).

Example 4. Let $n=1$, and for all $t, s \in \mathbb{R}^{+}$,

$$
\begin{gathered}
a_{1}(t)=\exp (\sin \pi t) \cos t, \\
b_{1}(t)=\pi \cos \pi t, \\
K_{11}(t, s)=\frac{\exp (\sin \pi t)}{(1+t+s)^{3} \exp (\sin \pi s)} .
\end{gathered}
$$

Then, for all $t \in \mathbb{R}^{+}$, we have $\beta_{1}(t)=\exp (\sin \pi t)$,

$$
\begin{gathered}
A_{1}(t)=\int_{0}^{t} \frac{a_{1}(s)}{\beta_{1}(s)} d s=\sin t, \\
A_{1}=\sup _{t \in \mathbb{R}^{+}}\left\{\left|A_{1}(t)\right|\right\}=1 \in(0,+\infty), \\
M_{1}=\int_{0}^{+\infty}\left(\int_{0}^{t}\left|K_{11}(t, s) \frac{\beta(s)}{\beta(t)}\right| d s\right) d t
\end{gathered}
$$

$$
\begin{aligned}
& =\int_{0}^{+\infty}\left(\int_{0}^{t} \frac{1}{(1+t+s)^{3}} d s\right) d t \\
& =\int_{0}^{+\infty} \frac{1}{2}\left[\frac{1}{(1+t)^{2}}-\frac{1}{(1+2 t)^{2}}\right] d t \\
& =\frac{1}{2} \int_{0}^{+\infty} \frac{1}{(1+t)^{2}} d t-\frac{1}{2} \int_{0}^{+\infty} \frac{1}{(1+2 t)^{2}} d t \\
& =\frac{1}{2}-\frac{1}{4}=\frac{1}{4} \in(0,1) .
\end{aligned}
$$

In addition, it is easy to see that

$$
\begin{aligned}
0<e^{-1} & =\liminf _{t \rightarrow+\infty} \beta_{1}(t) \\
& \leq \limsup _{t \rightarrow+\infty} \beta_{1}(t)=e<+\infty .
\end{aligned}
$$

Thus, by Theorem 3, we conclude that for every $c>1$, there exists a solution $x: \mathbb{R}^{+} \rightarrow \mathbb{R}$ for (2) such that

$$
x(t) \sim(c+\sin t) \exp (\sin \pi t), \quad t \longrightarrow+\infty .
$$

Remark 5. It is needed to note that in the above example, $(c+$ $\sin t) \exp (\sin \pi t)$ is not a solution to $(2)$.

Example 6. Consider the following system:

$$
\begin{aligned}
x_{i}^{\prime}(t)= & a_{i}(t)+b_{i}(t) x_{i}(t) \\
& +\sum_{j=1}^{2} \int_{0}^{t} K_{i j}(t, s) x_{j}(s) d s, \quad i=1,2,
\end{aligned}
$$

where

$$
\begin{gathered}
a_{1}(t)=\exp (\sin \pi t) \cos t, \\
a_{2}(t)=-\exp (\cos \pi t) \sin t, \\
b_{1}(t)=\pi \cos \pi t, \\
b_{2}(t)=-\pi \sin \pi t, \\
K_{i j}(t, s)=\frac{(-1)^{i+j} \exp (\sin \pi t)}{16(1+t+s)^{3} \exp (\sin \pi s)}
\end{gathered}
$$

for all $i, j=1,2$, and $t, s \in \mathbb{R}^{+}$. By a direct calculation, we get

$$
\begin{gathered}
\beta_{1}(t)=\exp (\sin \pi t), \\
\beta_{2}(t)=\exp (-1+\cos \pi t), \\
A_{1}(t)=\int_{0}^{t} \frac{a_{1}(s)}{\beta_{1}(s)} d s=\sin t, \quad A_{1}=1, \\
A_{2}(t)=\int_{0}^{t} \frac{a_{2}(s)}{\beta_{2}(s)} d s=(-1+\cos t) e, \quad t \in \mathbb{R}^{+}, A_{2}=2 e,
\end{gathered}
$$




$$
\begin{aligned}
M_{1} & =\sum_{j=1}^{2} \int_{0}^{+\infty}\left(\int_{0}^{t}\left|K_{1 j}(t, s) \frac{\beta_{j}(s)}{\beta_{1}(t)}\right| d s\right) d t \\
& \leq \frac{1+e}{64}<1, \\
M_{2} & =\sum_{j=1}^{2} \int_{0}^{+\infty}\left(\int_{0}^{t}\left|K_{2 j}(t, s) \frac{\beta_{j}(s)}{\beta_{2}(t)}\right| d s\right) d t \\
& \leq \frac{e+2 e^{3}}{64}<1 .
\end{aligned}
$$

Moreover, we have

$$
\begin{aligned}
0<e^{-1} & =\liminf _{t \rightarrow+\infty} \beta_{1}(t) \\
& \leq \limsup _{t \rightarrow+\infty} \beta_{1}(t)=e<+\infty, \\
0<e^{-2} & =\liminf _{t \rightarrow+\infty} \beta_{2}(t) \\
& \leq \limsup _{t \rightarrow+\infty} \beta_{2}(t)=1<+\infty .
\end{aligned}
$$

Then, by Theorem 3, for every $c=\left(c_{1}, c_{2}\right)^{T} \in \mathbb{R}^{2}$ with $c_{1}>1$ and $c_{2}>2 e$, there exists a solution $x=\left(x_{1}, x_{2}\right): \mathbb{R}^{+} \rightarrow \mathbb{R}^{2}$ of system (40) such that

$$
\begin{gathered}
x_{1}(t) \sim\left(c_{1}+\sin t\right) \exp (\sin \pi t), \quad t \longrightarrow+\infty, \\
x_{2}(t) \sim\left[c_{2}+(-1+\cos t) e\right] \exp (-1+\cos \pi t), \quad t \longrightarrow+\infty .
\end{gathered}
$$

\section{Conflict of Interests}

The authors declare that there is no conflict of interests regarding the publication of this paper.

\section{Acknowledgments}

The authors are grateful to Professor Diblík and two anonymous referees for their valuable suggestions and comments. In addition, Hui-Sheng Ding acknowledges support from the NSF of China (11101192), the Program for Cultivating Young Scientist of Jiangxi Province (20133BCB23009), and the NSF of Jiangxi Province; Yue-Wen Cheng acknowledges support from the Graduate Innovation Fund of Jiangxi Province.

\section{References}

[1] J. A. D. Applelby, I. Győri, and D. W. Reynolds, "On exact convergence rates for solutions of linear systems of Volterra difference equations," Journal of Difference Equations and Applications, vol. 12, no. 12, pp. 1257-1275, 2006.

[2] J. Diblík, E. Schmeidel, and M. Růžičková, "Existence of asymptotically periodic solutions of system of Volterra difference equations," Journal of Difference Equations and Applications, vol. 15, no. 11-12, pp. 1165-1177, 2009.
[3] J. Diblík, M. Růžičková, and E. Schmeidel, "Existence of asymptotically periodic solutions of scalar Volterra difference equations," Tatra Mountains Mathematical Publications, vol. 43, pp. 51-61, 2009.

[4] J. Diblík, E. Schmeidel, and M. Růžičková, "Asymptotically periodic solutions of Volterra system of difference equations," Computers \& Mathematics with Applications, vol. 59, no. 8, pp. 28542867, 2010.

[5] J. Diblík, E. Schmeidel, M. Růžičková, and M. Zbaszyniak, "Weighted asymptotically periodic solutions of linear Volterra difference equations," Abstract and Applied Analysis, vol. 2011, Article ID 370982, 14 pages, 2011.

[6] J. Diblík and E. Schmeidel, "On the existence of solutions of linear Volterra difference equations asymptotically equivalent to a given sequence," Applied Mathematics and Computation, vol. 218, no. 18, pp. 9310-9320, 2012.

[7] I. Győri and D. W. Reynolds, "On asymptotically periodic solutions of linear discrete Volterra equations," Fasciculi Mathematici, no. 44, pp. 53-67, 2010.

[8] W. Long and W.-H. Pan, "Asymptotically almost periodic solution to a class of Volterra difference equations," Advances in Difference Equations, vol. 2012, article 199, 2012.

[9] R. Medina, "Asymptotic behavior of solutions of Volterra difference equations with finite linear part," Nonlinear Studies, vol. 8, no. 1, pp. 87-95, 2001.

[10] R. Medina, "Asymptotic behavior of Volterra difference equations," Computers \& Mathematics with Applications, vol. 41, no. 5-6, pp. 679-687, 2001. 


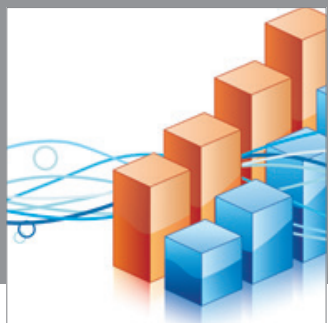

Advances in

Operations Research

mansans

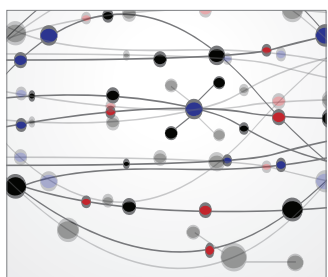

The Scientific World Journal
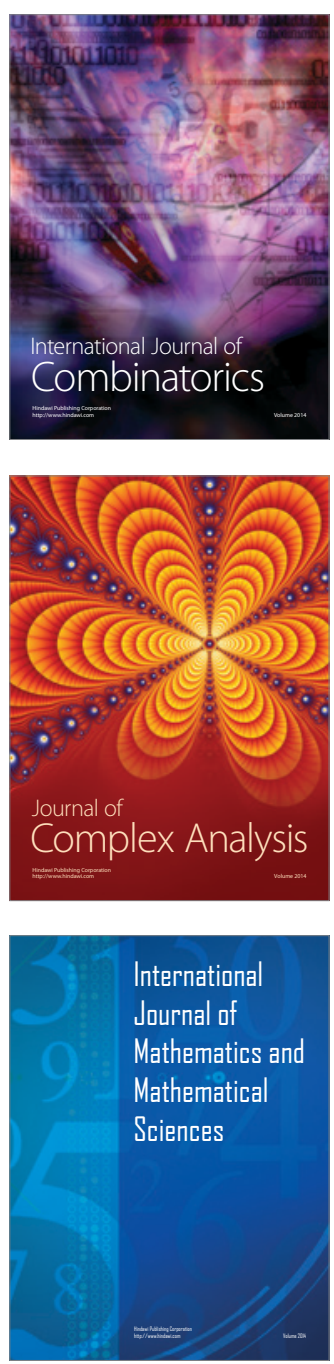
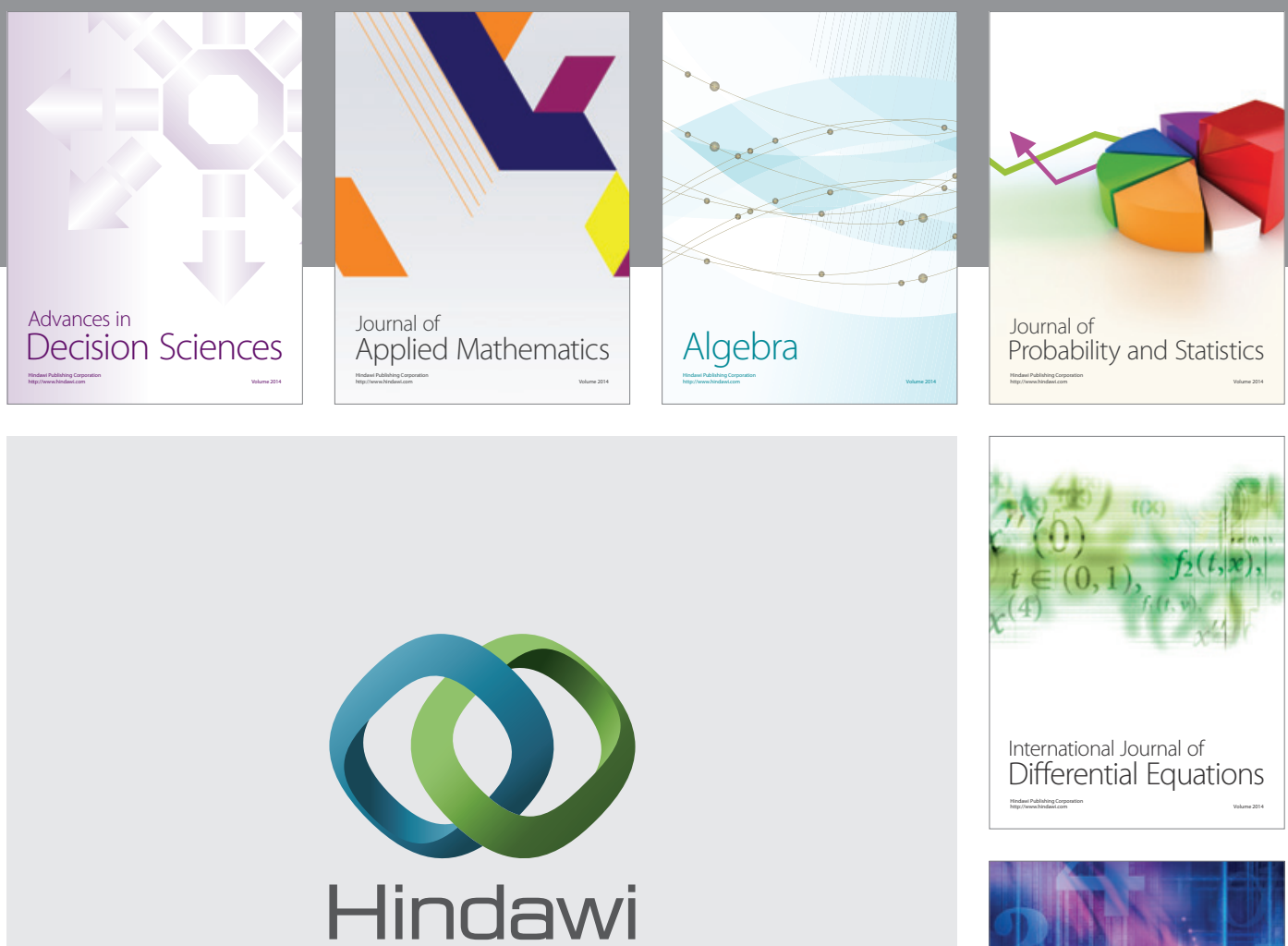

Submit your manuscripts at http://www.hindawi.com
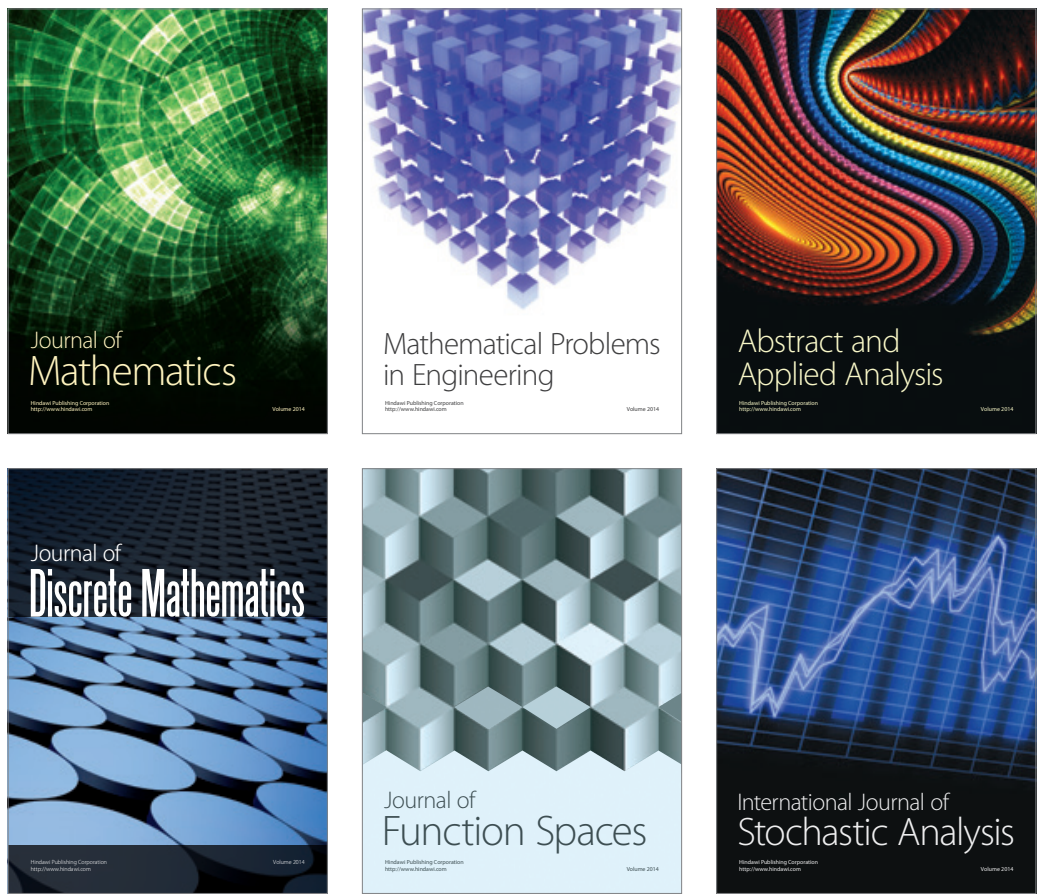

Journal of

Function Spaces

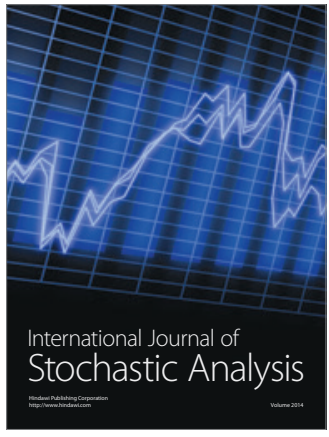

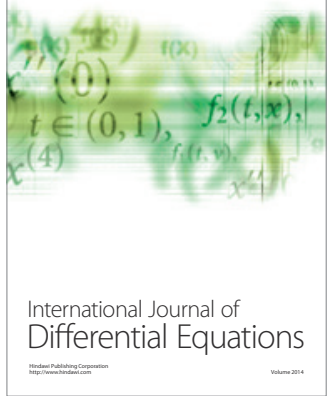
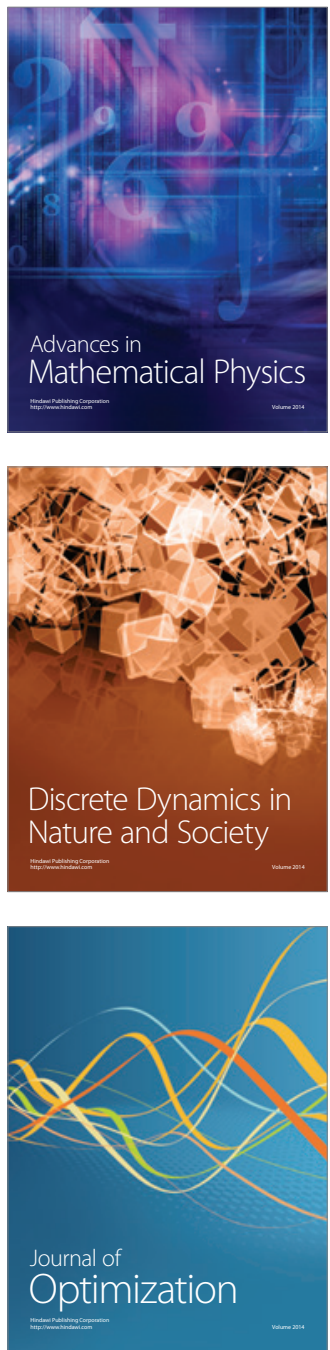